\title{
Verzerrtes Bild
}

_ Wie in jedem Beruf gibt es auch in der Physiotherapie ein paar, die es mit ihren Pflichten nicht allzu genau nehmen. Leider spielten ein paar davon die Hauptrolle in einer Studie, die kürzlich in einer Orthopädie-Fachzeitschrift veröffentlicht wurde (๑S. 33). Sie haben ein sehr schlechtes Bild hinterlassen, das die Autoren, zwei Orthopäden, auf die ganze Berufsgruppe projiziert haben: Physiotherapeuten schreiben keine Therapieberichte, obwohl erwünscht, und leiten keine Eigenübungen an, obwohl gefordert. Ihr Fazit: Das Kooperationsverhalten der Therapeuten sei massiv defizitär.

_ Bei ein paar schwarzen Schafen mag das stimmen. Doch die meisten von uns tun ihr Bestes, dass ihre Patienten schnell wieder fit

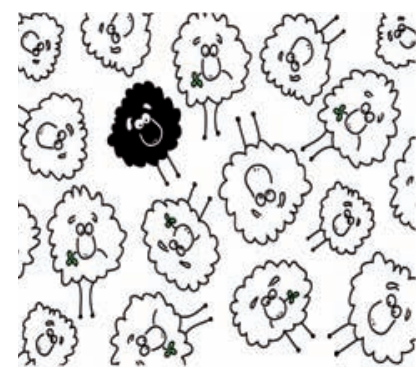
werden. Dazu gehört auch die konsequente Rücksprache mit Ärzten - schriftlich, per Telefon oder persönlich. In meiner therapeutischen Laufbahn war es selbstverständlich, mit Ärzten Hand in Hand zusammenzuarbeiten.

_ Als selbstverständlich erlebte ich auch, dass Physiotherapeuten Eigenübungen anleiten - obwohl es sicherlich einige Kollegen gibt, die sich nur schwer von „Hands on“ lösen können. Wie das wunderbar funktionieren kann, war übrigens neulich in einem fünfminütigen Beitrag des ZDF-Magazins „Volle Kanne“ $z u$ sehen (www.zdf.de/zdfmediathek > „1313966“): Philipp, neun Jahre alt und begeisterter Basketballer, hat Rückenschmerzen, weswegen ihn seine Eltern zum Doktor schicken. Schnitt. Man wartet auf den seriösen Arzt, der stirnrunzelnd Philipps Wirbelsäule beäugt. Doch stattdessen erscheint - quasi im filmischen Direktzugang - eine sympathische, kompetent wirkende Physiotherapeutin. Der Arzt wird in einem kurzen Satz abgehakt.

_ Die Therapeutin hält, was der erste Eindruck verspricht: Sie analysiert die Haltung, erklärt glasklar, wo das Problem liegt, therapiert kindgerecht, leitet ein Heimprogramm an und bezieht die Eltern mit ein. Am Ende des Beitrags ein Philipp, dem es viel besser geht. So stimmt das Bild.

Herzlichst, Ihr
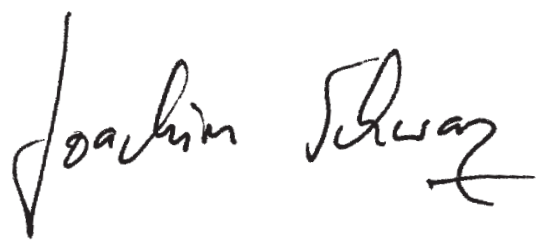

Ein schwarzes Schaf kann einen Schatten auf die ganze Herde werfen.

\section{ZU GEWIINNEN}

Für jede physiopraxis organisiert unsere gute Fee attraktive Gewinne für Sie. Möchten Sie den einen oder anderen ergattern, klicken Sie ins Internet unter: www.thieme.de/ physioonline > "physioexklusiv“. Und das gibt es in dieser Ausgabe zu gewinnen:

Kursplatz

1 Kurs nach Wahl im

Kölner Zentrum für

Frühbehandlung

Seite 59

Bücher

2-mal „Physiotherapie und Bewegungstraining für Hunde“

Seite 41

3-mal „Der ganz normale Wahnsinn“

Seite 44

und außerdem

2 MP3-Player

Seite 30

1 Airex-Matte 